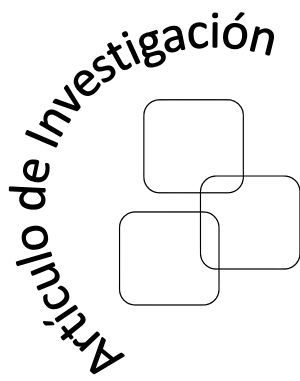

Julián Mauricio

Aguillón Pérez

Universidad Distrital

Francisco José de Caldas

Facultad de Ingeniería jmaguillonp@correo.udistrital.edu.co

Sergio Alejandro

Duarte Pacheco

Universidad Distrital

Francisco José de Caldas

Facultad de Ingeniería

saduartep@correo.udistrital.edu.co

Rodrigo Javier

Herrera García

Universidad Distrital

Francisco José de Caldas

Facultad de Ingeniería

rherrera@udistrital.edu.co

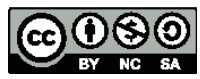

\section{Realce de candidatos a nódulo pulmonar en radiografías de tórax por medio de filtros de convergencia}

\section{Highlight of lung nodule candidates in chest radiographs by convergence filters}

\section{Resumen}

Este artículo presenta el desarrollo de un método para el realce de áreas sospechosas de nódulos pulmonares en radiografías de tórax. Los nódulos pulmonares son lesiones que se encuentran dentro de la región pulmonar y son los primeros indicadores de la presencia de un tumor cancerígeno en este órgano. En primera instancia, se implementa un método de segmentación automático. Luego, para el realce de los candidatos, se usan filtros de convergencia que evalúan el grado en el que converge el área circundante al píxel de interés, asumiendo que los posibles candidatos a nódulos presentan forma circular. En este trabajo se implementan cuatro filtros de convergencia: Moneda, Iris, Anillo Adaptativo y Banda Deslizante. Los filtros de moneda y de anillo adaptativo obtuvieron mejores resultados en cuanto a detección y número de candidatos por imagen, imágenes pertenecientes a la base de datos de la JSRT (Japanese Society of Radiological Technology). $\mathrm{Al}$ aplicar la técnica de resta contralateral junto con los filtros basados en características geométricas tales como área, redondez y excentricidad, se logró disminuir el número de candidatos.

Palabras claves: Radiografía de tórax, nódulos pulmonares, segmentación, filtros de convergencia.

\section{Abstract}

This paper describes the development of a method that highlights suspicious areas of lung nodules on chest radiographs. Lung nodules are lesions found in the lung region, which are the first indicators of the presence of cancerous tumors. First, an automatic segmentation method is implemented. Next, to highlight the candidates, convergence filters were used in order to evaluate the degree in which the surrounding area converged to the pixel of interest, assuming a circular shape for potential nodule candidates. Four convergence filters were implemented: Coin filter, Iris filter, Adaptive Ring filter and Sliding Band filter. Coin and adaptive ring filters obtained better results in terms of detection and number of candidates per image, images from the database of the JSRT (Japanese Society of Radiological Technology). Applying the contralateral subtraction technique together with filters based on geometric features such as areas, roundness and eccentricity, it was possible to reduce the number of candidates.

Key words: Chest radiographs, lung nodules, segmentation, convergence index filters. 


\section{Introducción}

El cáncer de pulmón es la causa más frecuente de mortalidad por cáncer en el mundo. Es, además, uno de los cánceres con peor pronóstico ya que su detección suele darse en etapa avanzada. Para seleccionar el tratamiento es fundamental hacer una estadificación adecuada, ya que una detección en estado de nódulo pulmonar permitirá una intervención terapéutica que traerá consigo un mejor pronóstico. El diagnóstico de los nódulos pulmonares es además importante para la detección de metástasis pulmonares en tumores extratorácicos. Por lo tanto, aún continúa siendo un problema clínico de primera magnitud [18], siendo la radiografía de tórax la herramienta básica de detección [1] [5]. Una ayuda que con el paso del tiempo se ha hecho cada vez más relevante en el diagnóstico médico es el CAD (Computer Aided Diagnosis) [21].

En la búsqueda de regiones pulmonares, la segmentación de los pulmones y la detección de nódulos son las tareas principales. Los nódulos aparecen en las radiografías como objetos relativamente circulares, iluminados y de bajo contraste dentro de la región pulmonar. La dificultad para los CAD es distinguir nódulos verdaderos de objetos superpuestos como vasos y costillas. La primera etapa de la detección de nódulos es encontrar candidatos. Luego se hace necesario eliminar el mayor número de candidatos que son nódulos, evitando sacrificar verdaderos positivos [21].

La segmentación pulmonar es la primera etapa para desarrollar un algoritmo de detección de nódulos. La razón principal es restringir la región de interés para así solo trabajar en zonas con mayor probabilidad de encontrar estas anomalías. Usualmente se emplean sistemas basados en reglas, como por ejemplo el uso del histograma para encontrar un valor de umbralización, el uso del gradiente de Sobel para remover el ruido adjunto a los pulmones [13]. Otro enfoque de segmentación es el que usa aprendizaje supervisado, como por ejemplo el uso del modelo de forma activa ASM (Active Shape Model), la apariencia activa AAM (Active Appareance Model) y la clasificación de píxeles PC [20].

En la detección de nódulos existen distintos enfoques como el modelo de contorno activo, llamado serpiente (snake), basado en Wavelets [24] [23]. La resta contralateral C-Sub (Contralateral Substraction) es una técnica que mejora la visibilidad de los nódulos por la sustracción de la imagen espejo invertida de la imagen original, usando métodos de registro global y local [8] [7]. El uso de redes neuronales es otro enfoque en la detección de nódulos; por ejemplo el uso de las redes de tipo realimentado permite un uso eficiente del conocimiento a priori de la forma de los nódulos y las estructuras del segundo plano [2]. Una ayuda adicional es la implementación de algoritmos genéticos si se usa ajuste de plantilla (Template Matching), al identificar las posiciones adecuadas y la forma de las imágenes de referencia [6].

Para el realce de nódulos pulmonares se usan distintos tipos de filtros. Por ejemplo, los filtros LoG (Laplacian of Gaussian) se usan con una adecuada selección de la desviación estándar del núcleo [15].

En la tarea de reducir falsos positivos se han estudiado herramientas como SVM (Máquinas de Soporte Vectorial) que permite el reconocimiento entrenado de nódulos verdaderos. Las SVM están basadas en la teoría de aprendizaje estadístico y en la minimización de riesgo 
estructural, obteniendo la mayor habilidad de generalización mediante la consecución de un balance óptimo entre la complejidad del modelo y la capacidad de aprendizaje [10][25].

En este artículo se describe un método de realce de nódulos pulmonares en radiografía de tórax. En la sección de metodología, se describirán los algoritmos de segmentación pulmonar y de realce de candidatos a nódulo. Luego se presentarán y discutirán los respectivos obtenidos y por último se harán las conclusiones correspondientes.

\section{Metodología}

En esta sección, se describe la herramienta de software desarrollada para el realce de candidatos a nódulo pulmonar en radiografías de tórax. La primera etapa describe la segmentación pulmonar precedida de una etapa de preprocesamiento. En seguida, se explica la técnica para la detección de los nódulos pulmonares, por medio de los filtros de convergencia. Al final se incluye un método de clasificación basado en características geométricas. En la Figura 1, se muestra la metodología general usada en este documento.

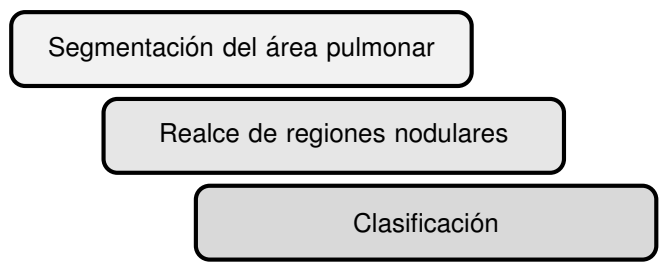

Figura 1. Metodología general para la detección de candidatos a nódulo

\subsection{Segmentación pulmonar}

La segmentación pulmonar se realiza para restringir la detección de los nódulos a la región pulmonar. En este trabajo, se aplicó el algoritmo presentado en la Figura 2. El preprocesamiento es la primera etapa del método. La aproximación al área pulmonar basada en la mejora de contraste local 2, la binarización por Otsu y la eliminación de regiones que no pertenecen a la región de interés forman parte de la segunda etapa. Con la mejora de contrastes locales 1 y 2; la aplicación del filtro de Gabor, que son filtros direccionales aplicados a cada pulmón y la binarización por Otsu corresponden a la aproximación de los bordes externos de los pulmones, siendo esta la tercera etapa. Por último, se realiza la unión de los resultados de la segunda y tercera etapa y se realiza un postprocesamiento.

\subsubsection{Preprocesamiento}

La primera etapa consiste en hacer preprocesamiento sobre las imágenes. En primer lugar, se redimensionan las imágenes de $2048 \times 2048$ píxeles a $512 \times 512$ píxeles con el fin re- 


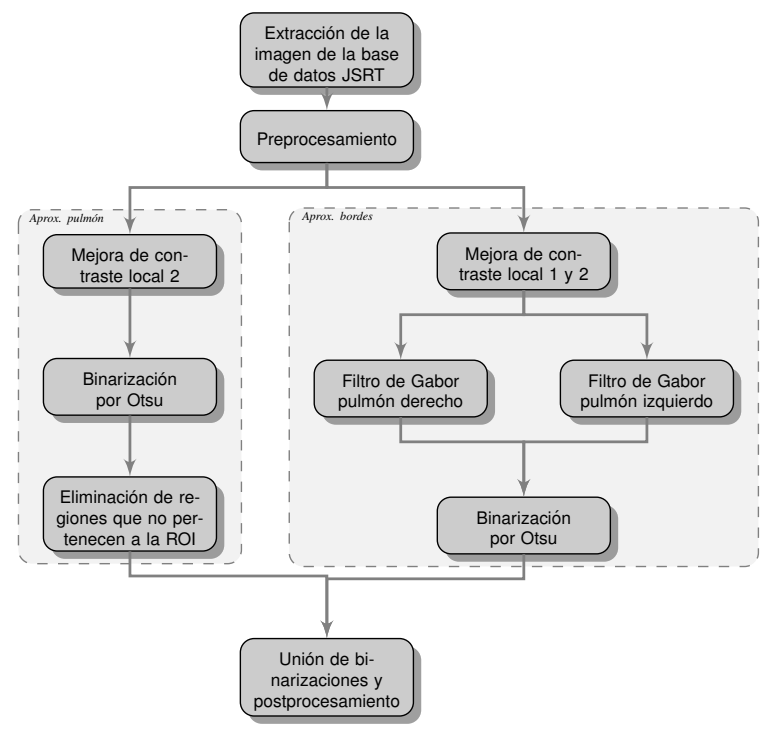

Figura 2. Diagrama de la segmentación del área pulmonar propuesta

ducir el tiempo de procesamiento. Como las imágenes de radiografías de tórax presentan un amplio rango de iluminación pero a la vez bajo contraste, se hace necesario normalizar los histogramas. Para lograr esto, se usó la Ecualización Adaptativa del Histograma por Contraste Limitado CLAHE (Contrast Limited Adaptive Histogram Equalization) [11]. El método CLAHE permite la reducción de ruido típica de la ecualización del histograma adaptativa, ya que limita la pendiente de los niveles de gris recortando el histograma a un determinado valor. Los píxeles que son recortados se redistribuyen igualmente para mantener la totalidad del histograma intacto.

\subsubsection{Contrastes locales}

Pretenden mejorar el contraste local de las imágenes (entendido como la diferencia relativa en intensidad entre un punto de una imagen y sus alrededores), se busca que el área pulmonar presente una uniformidad en su contraste. Se define la mejora del contraste local 1 por medio de la siguiente ecuación:

$$
Y(x, y, k)=\frac{I(x, y)-\mu_{l}(k)}{\sigma_{g}}
$$

donde $I(x, y)$ es la imagen de entrada, $Y(x, y, k)$ es la imagen de salida $\mu_{l}(k)$ es la media local y $\sigma_{g}$ es la desviación estándar global. Aquí $k=1 \cdots \mathrm{N}$, donde $\mathrm{N}$ es el número de regiones locales. La mejora del contraste local 2 usa la desviación estándar local $\sigma_{l}(k)$ en lugar de la global [17], como se observa en la siguiente ecuación: 


$$
Y(x, y, k)=\frac{I(x, y)-\mu_{l}(k)}{\sigma_{l}(k)}
$$

Al aplicar la mejora del contraste local 2 y binarizando, se obtiene una aproximación inicial al área pulmonar. Igualmente, como se observó en la Figura 2, al aplicar secuencialmente la mejora del contraste local 1 con la mejora del contraste local 2 se asegura un mejor realce en los bordes externos de los pulmones.

\subsubsection{Binarización por Otsu}

Como se ha mencionado antes, una primera aproximación al área pulmonar se obtiene luego de binarizar la imagen. El método de binarización usado es el de Otsu que básicamente realiza una búsqueda exhaustiva del umbral óptimo al maximizar la varianza entre las clases que forman la imagen (fondo y objeto) [9], teniendo en cuenta que una imagen se puede asumir como una función bidimensional en intensidad de niveles de grises que contiene el número total de píxeles y considerando el rango en el que están los niveles de grises.

\subsubsection{Eliminación de regiones que no pertenecen a la región de interés}

Cuando se tiene la aproximación inicial a la región pulmonar y al binarizar por medio del método de Otsu, por lo general quedan regiones adicionales a los dos pulmones que se hace necesario eliminar. Al observar una imagen de radiografía, hay regiones que en los bordes de la imagen se destacan del fondo. La eliminación de las regiones que tocan los bordes se hace por medio de un algoritmo de crecimiento. Luego de que se tienen los bordes limpios de regiones indeseadas, se etiquetan las regiones restantes donde las dos con mayor área harán referencia a los pulmones.

Esta segmentación presenta los pulmones separados de ellos mismos y de otras estructuras de la imagen como la tráquea o las partes externas del tórax presentes en la radiografía, pero que hacen parte del fondo de la imagen. Sin embargo, en la mayoría de las imágenes, el área segmentada es menor que el área real de los pulmones y hay una pérdida generalizada en los bordes externos debido al bajo contraste con el fondo de la imagen.

\subsubsection{Filtros de Gabor}

Para resaltar las costillas y los bordes externos se requiere el uso de filtros direccionales como los filtros de Gabor. Un filtro de Gabor en 2-D, se describe por medio de la siguiente ecuación [14]:

$$
g(x, y)=\exp \left(-\frac{1}{2}\left[\frac{x_{\theta}^{2}}{\sigma_{x}^{2}}+\frac{y_{\theta}^{2}}{\sigma_{y}^{2}}\right]\right) \cdot \cos \left(\frac{2 \pi \cdot x_{\theta}}{T}\right)
$$


donde las variables $\left(x_{\theta}, y_{\theta}\right)$ proceden de efectuar un giro sobre las coordenadas iniciales $(x, y)$ :

$$
\left(\begin{array}{l}
x_{\theta} \\
y_{\theta}
\end{array}\right)=\left[\begin{array}{cc}
\cos (\theta) & \sin (\theta) \\
-\sin (\theta) & \cos (\theta)
\end{array}\right]\left(\begin{array}{l}
x \\
y
\end{array}\right)
$$

Puede verse el filtro como el producto de una gaussiana modulada por una componente sinusoidal, donde $\theta$ es la orientación y $T$ es el período. Al verse el filtro en frecuencia va a ser pasabajo en una dirección y pasabanda en la perpendicular. Por lo tanto la salida tenderá a conservar solo las líneas orientadas en dirección $\theta$ y que estén separadas una distancia $T$.

Un replanteo de parámetros de los filtros de Gabor, se destaca a continuación [12]:

$$
g_{\lambda, \sigma, \theta, \varphi}=\exp \left(\frac{x_{\theta}+\left(\gamma \cdot y_{\theta}\right)^{2}}{2 \sigma^{2}}\right) \cos \left(2 \pi \frac{x_{\theta}}{\lambda}+\varphi\right)
$$

Donde $\gamma$ es la relación de aspecto espacial, que determina la elipticidad de la respuesta al impulso. La desviación estándar $\sigma$ del factor gaussiano determina el tamaño de la ventana. El parámetro $\lambda$ es la longitud de onda, siendo $1 / \lambda$ la frecuencia espacial del factor coseno. La relación $\sigma / \lambda$ determina el ancho de banda $(b)$ de la frecuencia espacial. El parámetro $\varphi$ es el desplazamiento de fase que determina la simetría de la función de Gabor con respecto al origen: para $\varphi=0$ y $\varphi=\pi$ es simétrica o par, y para $\varphi=\pi / 2$ y $\varphi=-\pi / 2$ es antisimétrica o impar; para los otros casos son mezclas asimétricas.

Este filtro de Gabor es aplicado sobre las imágenes luego de ser mejoradas por medio de los contrastes locales 1 y 2 . Seguido de esto se binariza por medio de Otsu, y se procede a realizar la intersección con la aproximación del área pulmonar. Dicha área se unirá con la aproximación del área pulmonar obtenida anteriormente y así se obtendrá la segmentación final, como se observa en la Figura 3. Usando como base la plantilla proporcionada por la base de datos SCR se puede observar la intersección de la segmentación con el método realizado con la referencia estándar.

\subsection{Realce de regiones candidatas a nódulo}

A causa de la baja intensidad de los rayos X, las radiografías torácicas presentan bajo contraste y alto ruido, por lo que los nódulos pulmonares relativamente no se evidencian en la imagen.

Para realzar dichos nódulos, se implementaron filtros de convergencia, a causa de que se asume que los nódulos pulmonares tienen forma circular y estos filtros son efectivos para el realce de este tipo de regiones. Debido a que el número de candidatos a nódulo aún es elevado, la técnica de resta contralateral elimina un número mayor de estos candidatos. 


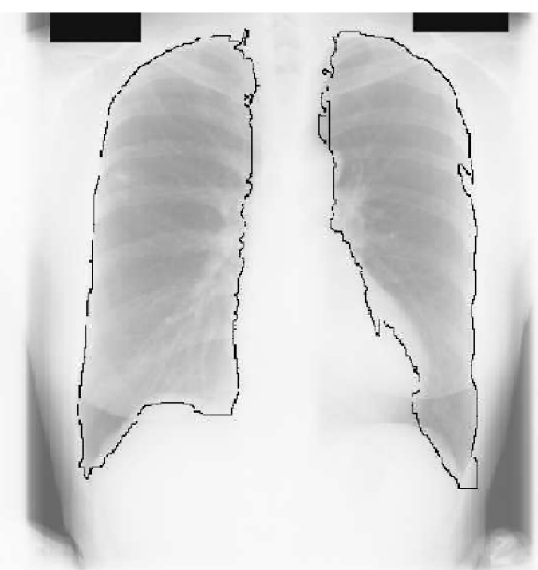

(a)

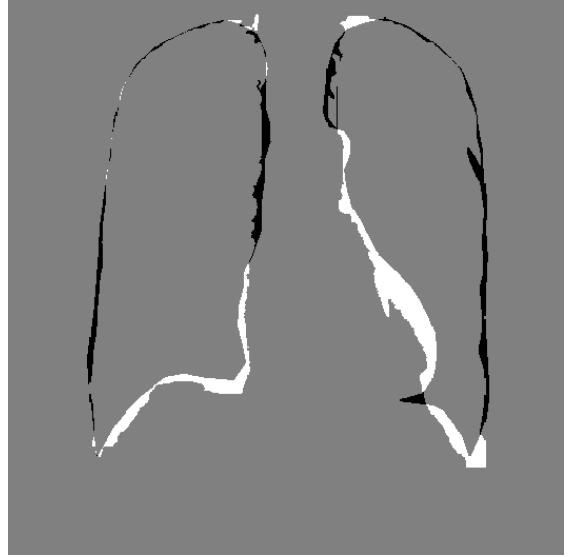

(b)

Figura 3. Resultado de la segmentación aplicada al archivo JPCLN034: (a) muestra el área pulmonar segmentada superpuesta sobre la imagen original y (b) muestra la comparación con la referencia estándar de la base de datos SCR.

Por último, filtrando las regiones por ciertas características geométricas como área, redondez y excentricidad, se logra aún más reducir el número de candidatos sin sacrificar nódulos verdaderos. En la Figura 4 se muestra el diagrama del procedimiento efectuado.

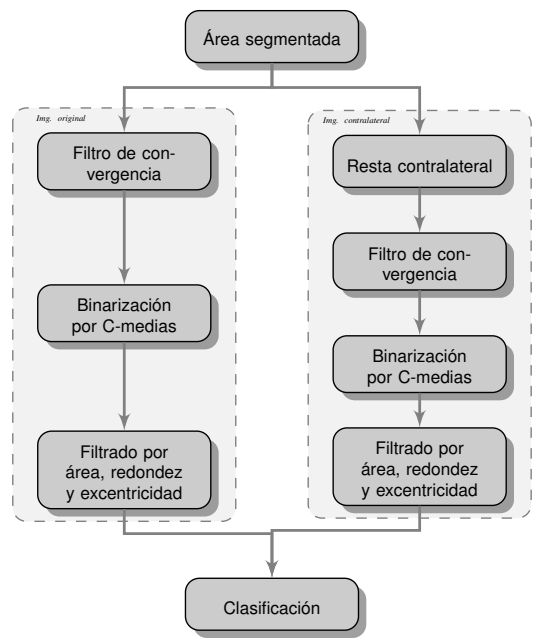

Figura 4. Diagrama del método de realce y clasificación de candidatos a nódulo pulmonar

\subsubsection{Filtros de convergencia}

Como se ha mencionado, los nódulos pulmonares presentan forma aproximadamente circular, destacándose del fondo de una imagen digital como montañas cónicas. Sus líneas de intensidad se pueden modelar como círculos concéntricos, llamados regiones convexas. Los vectores gradientes de la región convexa apuntan hacia su cima. De esta forma es que las 
regiones convexas se pueden detectar, ya que son las regiones donde los vectores gradientes convergen a un mismo centro dentro de un área local o región de apoyo. Un método para evaluar el grado de convergencia consiste en $N$ semirrectas radiando desde el píxel de interés $P$. La orientación de los vectores gradientes de la imagen en relación a las columnas $G_{c}$ y las filas $G_{f}$ para cada píxel en la imagen original se definen así [4]:

$$
\alpha(x, y)=\arctan \frac{G_{c}(x, y)}{G_{f}(x, y)}
$$

El ángulo de cada semirrecta con respecto al píxel de interés $\theta_{i}$ se define por medio de la siguiente ecuación:

$$
\theta_{i}=\frac{2 \pi}{N}(i-1)
$$

donde $N$ es el número de semirrectas donde se ha evaluado la convergencia, de acuerdo a sus direcciones radiales. En este caso se usaron 8 rectas debido a que permiten garantizar los puntos necesarios para detectar la mayor convergencia posible dentro de la región de apoyo del filtro, además que el costo computacional es razonablemente bajo.

El grado de convergencia de los vectores gradientes sobre una semirrecta se puede expresar por medio de la siguiente ecuación [22]:

$$
c_{i}=\frac{1}{R} \sum_{j=1}^{R} \cos \left(\theta_{j}\right)
$$

donde $R$ es el número de píxeles sobre la $i$-ésima semirrecta y $\cos \left(\theta_{j}\right)$ es la orientación del j-ésimo vector gradiente de $P$ con respecto a la orientación de la $i$-ésima semirrecta. $\mathrm{El} \cos (\theta)$ es equivalente a la convergencia de cada píxel en la imagen a tratar y se define como Índice de Convergencia (IC) como se muestra a continuación:

$$
\mathrm{IC}(x, y, i)=\cos \left(\theta_{i}-\alpha(x, y)\right)
$$

La convergencia global se obtiene mediante la suma de todas las convergencias individuales, dada por la ecuación 9, dentro de la zona de soporte específica de cada filtro. Se implementaron cuatro filtros de convergencia que varían de acuerdo a la región de apoyo: Filtro de Moneda (FM), Filtro de Iris (FI), Filtro de Anillo Adaptativo (FAA) y Filtro de Banda Deslizante (FBD).

El Filtro de Moneda (FM) es el filtro de convergencia más elemental de todos, al tener una región de apoyo fija circular (Figura 5a). Se define por medio de la siguiente ecuación: 


$$
\operatorname{FM}(x, y)=\operatorname{máx}_{0 \leq r \leq R_{\text {máx }}} \frac{1}{N \cdot r} \sum_{i=0}^{N-1} \sum_{m=1}^{r} \operatorname{IC}(x, y, i)
$$

donde IC es el índice de convergencia y $N$ es el número de semirrectas a evaluar que conforman la región de apoyo y $R_{\text {máx }}$ es el radio máximo para la región de apoyo.

El Filtro de Iris (FI) es un filtro de moneda pero adaptativo, lo que significa que no tiene una región de apoyo estrictamente circular (Figura 5b). El FI maximiza la convergencia de cada radio independientemente adaptando el radio de la región de apoyo para cada semirrecta. El filtro se puede modelar por medio de la siguiente ecuación:

$$
\mathrm{FI}(x, y)=\frac{1}{N} \sum_{i=0}^{N-1}\left[\operatorname{máx}_{0 \leq r \leq R_{\text {máx }}} \frac{1}{r} \sum_{m=1}^{r} \operatorname{IC}(x, y, i)\right]
$$

El Filtro de Anillo Adaptativo (FAA) tiene una región de apoyo en forma de anillo y cambia el radio adaptativamente (Figura 5c). La salida del filtro se puede calcular por medio de la siguiente ecuación:

$$
\operatorname{FAA}(x, y)=\operatorname{máx}_{0 \leq r \leq R_{\text {máx }}} \frac{1}{N \cdot d} \sum_{i=0}^{N-1} \sum_{m=r-d / 2}^{r+d / 2} \mathrm{IC}(x, y, i)
$$

donde $d$ es una constante que corresponde al ancho o grosor del anillo en número de píxeles.

El Filtro de Banda Deslizante (FBD) básicamente une las características del FI con las del FAA, al definir una región de apoyo con una banda de ancho fijo y un radio adaptable a cada dirección (Figura 5d). De esta forma, se permite maximizar el grado de convergencia en cada punto. El FBD se calcula por medio de la siguiente ecuación:

$$
\operatorname{FBD}(x, y)=\frac{1}{N} \sum_{i=0}^{N-1} \operatorname{máx}_{R_{\min } \leq r \leq R_{\text {máx }}}\left[\frac{1}{d} \sum_{m=r-d / 2}^{r+d / 2} \mathrm{IC}(x, y, i)\right]
$$

Como se ha mencionado, el FBD se deriva de la estimación del FI y del FAA. De acuerdo a la ecuación, el ancho $d$ se mueve entre un radio mínimo y uno máximo ( $R_{\text {mín }}$ y $\left.R_{\text {máx }}\right)$.

En la Figura 6 se puede apreciar el comportamiento de los filtros de convergencia aplicados en las imágenes de radiografía de tórax con los pulmones segmentados con la base de datos SCR y usando un radio de 15 píxeles (Figura 6a). Con el FM se puede apreciar el realce de algunas áreas dentro de los pulmones y el realce del borde de los mismos (Figura 6b). Esto es debido a que el filtro busca el mayor índice de convergencia para cada uno de los radios y 


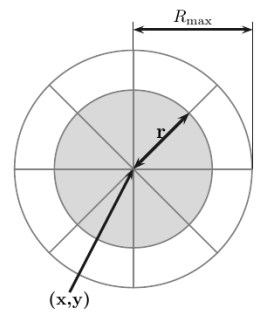

(a)

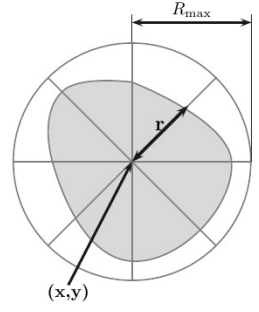

(b)

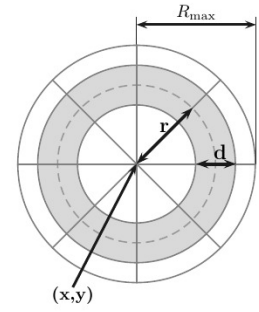

(c)

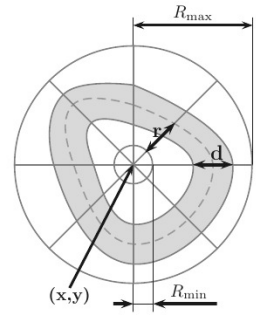

(d)

Figura 5. Filtros de convergencia: (a) Filtro de Moneda (FM), (b) Filtro de Iris (FI), (c) Filtro de Anillo Adaptativo (FAA) y (d) Filtro de Banda Deslizante (FBD)

es asignado al píxel de interés. Al comparar el resultado del FM con el FI, se puede observar que el FI genera una imagen más borrosa o difuminada, debido a que este filtro busca el mayor índice de convergencia para cada una de las direcciones y el mayor índice de todas es asignado al píxel de interés, presentando una forma irregular (Figura 6c). El FAA presenta un resultado parecido al FM, pero debido a la presencia del ancho $d$ las áreas resaltadas son más gruesas (Figura 6d). Los resultados del FBD son los que presentan áreas más difuminadas o desvanecidas entre todos los filtros de convergencia, siendo el comportamiento más parecido al FI pero debido al ancho $d$ hay áreas resaltadas más gruesas y borrosas (Figura 6e).

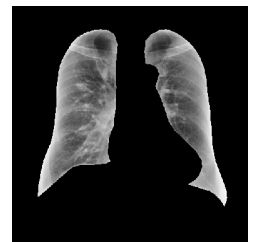

(a)

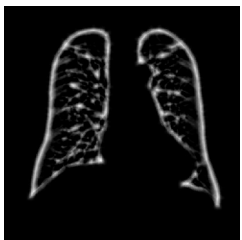

(b)

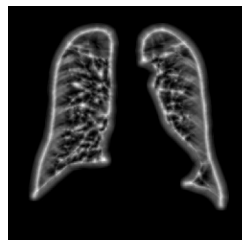

(c)

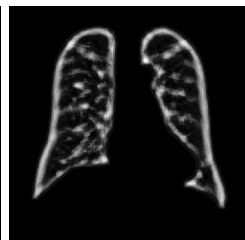

(d)

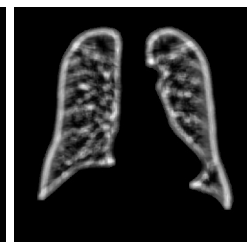

(e)

Figura 6. Aplicación de los filtros de convergencia: (a) Imagen a filtrar, (b) resultado del filtro de moneda, (c) resultado del filtro de iris, (d) resultado del filtro de anillo adaptativo y (e) resultado del filtro de banda deslizante

\subsubsection{Segmentación por C-Medias}

Al aplicar los filtros de convergencia para el realce de candidatos a nódulo, se segmentan dichos candidatos por medio de binarización, con el fin de aislarlos y luego sobreponerlos sobre la radiografía de tórax. Debido a que los resultados de los filtros de convergencia presentan estructuras dentro de la región pulmonar que están unidas al borde del pulmón y a otras regiones como costillas, se hace necesario buscar un método de segmentación que logre el mayor número de candidatos aislados dentro de la región pulmonar.

El método difuso de C-Medias FCM (Fuzzy C-Means) mostró ser eficiente en este aspecto. Básicamente la segmentación por FCM propone una pertenencia difusa que asigna un grado de pertenencia para cada clase. La formación de nuevos agrupamientos es posible con el monitoreo de los conjuntos de datos que tienen valores cercanos a las clases existentes. El método de FCM busca minimizar la siguiente ecuación [3]: 


$$
Q=\sum_{j=1}^{n} \sum_{j=1}^{C} U_{i j}^{m}\left\|v_{i}-\mu_{j}\right\|^{2} ; \quad 1 \leq m<\infty
$$

Donde $n$ es el número de muestras y $C$ es el número de agrupamientos; $\mu_{i}$ es el centro $d$-dimensional del agrupamiento; $m \in[1, \infty]$ es un exponente de ponderación y cualquier número real mayor a $1 ; v_{i}$ es el $i$-ésimo del dato medido $d$-dimensional; $\mathrm{y}\|\cdot\|$ es una norma que expresa la similaridad entre algún dato medido y el centro del agrupamiento. La matriz de pertenencia u satisface la siguiente condición:

$$
\sum_{i=1}^{C} \mu_{i j}=1, \quad \forall j=1, \ldots, n
$$

La pertenencia y los centros del agrupamiento son actualizados por medio de las siguientes relaciones respectivas:

$$
\mu_{i j}^{m}=\frac{1}{\sum_{k=1}^{C}\left(\frac{\left\|v_{i}-\mu_{i}\right\|}{\left\|v_{i}-\mu_{k}\right\|}\right)^{\frac{2}{m-1}}}
$$

$\mathrm{y}$

$$
\mu_{j}=\frac{\sum_{i=1}^{n} \mu_{i j} \cdot v_{i}}{\sum_{i=1}^{n} \mu_{i j}}
$$

Los centroides son la media de las distancia de los datos ponderados por su pertenencia y normalizados a la pertenencia total. Se puede observar que el grado de pertenencia es inversamente proporcional a la distancia del centro del agrupamiento.

La Figura 7 muestra el resultado al aplicar el algoritmo FCM con dos agrupamientos (Figura 7a) y con tres agrupamientos (Figura 7b) a una imagen con FM de 15 píxeles y segmentada con la base de datos SCR. Con dos agrupamientos muchas de las regiones resaltadas se unen con las costillas y los bordes externos. Con tres agrupamientos se permite descartar regiones que no son consideradas ni como fondo de la imagen ni como posible nódulo, como las costillas, clavículas o vasos. Este procedimiento es realizado uniendo los dos agrupamientos que se descartan de la segmentación, generando una imagen binarizada solo con los posibles candidatos a nódulos (Figura 7c). La implementación de más de tres agrupamientos implica mayor costo computacional y una posible pérdida de nódulos verdaderos. 


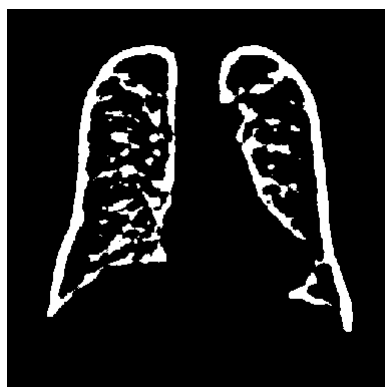

(a)

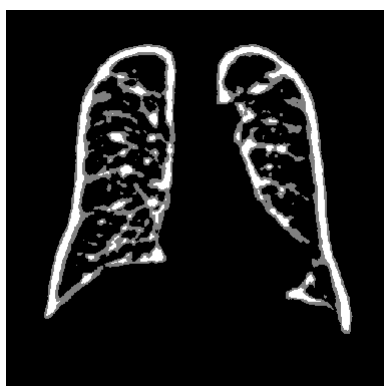

(b)

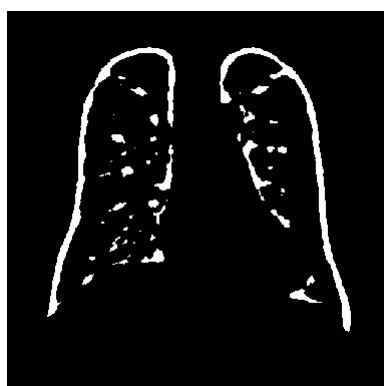

(c)

Figura 7. Aplicación de la segmentación FCM: (a) FCM con dos agrupamientos, (b) FCM con tres agrupamientos y (c) binarización de (b) uniendo dos agrupamientos.

\subsubsection{Resta Contralateral}

La técnica de resta contralateral ha sido usada para ayudar a radiólogos en la detección de anomalías asimétricas tales como nódulos pulmonares con una sola radiografía de tórax [19]. En este caso, su utilidad se validará en la reducción de candidatos a nódulos pulmonares, debido a que permite la eliminación de estructuras esqueléticas simétricas como las costillas dentro de la zona pulmonar. La imagen de resta contralateral se obtiene restando a la imagen original, su imagen invertida espejo y así se resaltan las anomalías o lesiones por dicha eliminación de estructuras.

Para realizar este método como primer paso se normalizaron los niveles de intensidad de la imagen de entrada en un rango de 0 a 1 . En la Figura 8 se muestra un ejemplo del desarrollo sobre una imagen de esta técnica: la imagen original (Figura 8a) se invirtió obteniéndose la imagen espejo (Figura 8b), realizándose la resta de la imagen original y la espejo (Figura 8c) y a cuyo resultado se le aplicá el filtro de moneda con radio de 15 píxeles (Figura 8d).

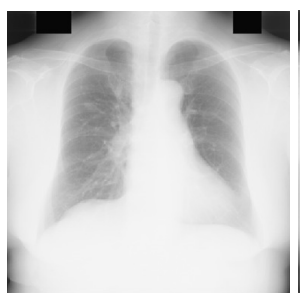

(a)

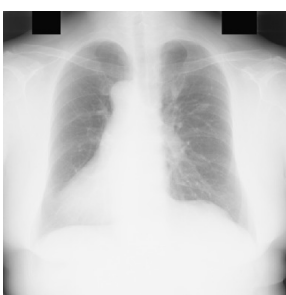

(b)

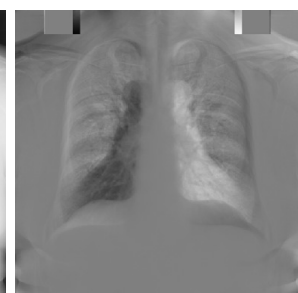

(c)

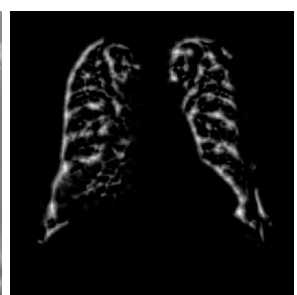

(d)

Figura 8. Desarrollo de la resta contralateral en el archivo JPCLN085: (a) Imagen original, (b) imagen invertida o espejo, (c) resultado de la aplicación de la resta contralateral y (d) aplicación a (c) del filtro de moneda.

\subsubsection{Clasificación}

De acuerdo con la Figura 4, luego de aplicar los filtros de convergencia y la segmentación por FCM tanto a la imagen original como a la de resta contralateral, el número de candidatos a nódulo en promedio por imagen aún es alto. Para reducir el número de candidatos y partiendo 
de la idea de que los nódulos tienen forma circular u ovalada y de estar definidos por su tamaño, se desarrollaron unos filtros que miden tres características geométricas: área, redondez y excentricidad. Estas características fueron seleccionadas de acuerdo con un conocimiento previo de la forma de los nódulos tomando en consideración a la mitad de las imágenes para dicho estudio.

Al clasificar por área, redondez y excentricidad los resultados de la segmentación por FCM en la imagen original y en la de resta contralateral, se realiza la intersección de estas dos imágenes binarizadas. En esta intersección se eliminan las regiones que no coinciden en ambas imágenes, resultando finalmente una reducción considerable de candidatos a nódulo como se observa en la Figura 9, donde el asterisco rojo indica el nódulo verdadero.

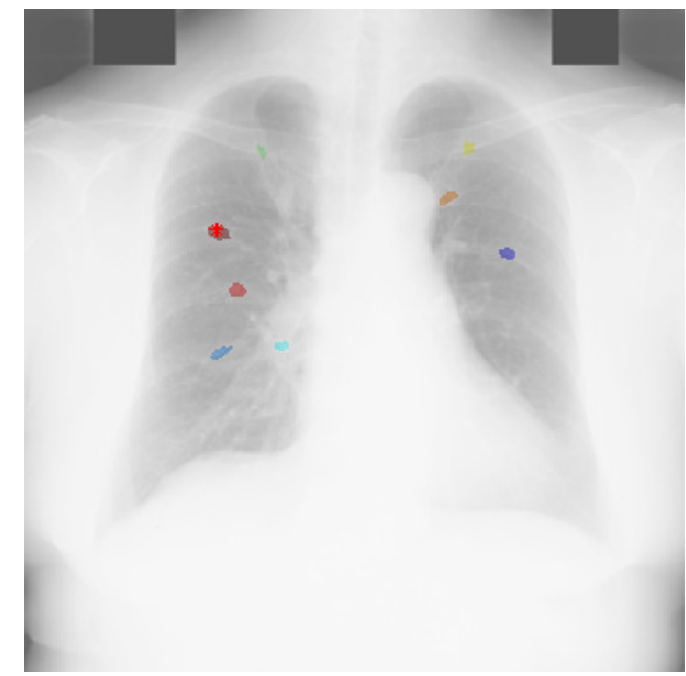

Figura 9. Resultado final del algoritmo de realce de candidatos a nódulo pulmonar

\section{Resultados}

Para el desarrollo de este trabajo se contó con la base de datos de la JSRT [16](Japanese Society of Radiological Technology) que cuenta con 154 imágenes de radiografía de tórax con presencia de un nódulo pulmonar solitario y 93 sin lesiones. Todas las imágenes tienen un tamaño de $2048 \times 2048$ píxeles, equivalente a $0,175 \mathrm{~mm}$ por píxel y 12 bits de profundidad. Las 154 imágenes con nódulo fueron clasificadas en cinco tipos de sutileza, iniciando del nivel 1 (el más sutil) hasta el 5 que significa que la presencia del nódulo es obvia en la imagen. Para efectos de este trabajo, la evaluación del método de realce se hizo con los niveles 3, 4 y 5 de sutileza y con nódulos que se encuentran dentro de la región pulmonar, lo que corresponde a 98 imágenes. Se debe destacar que la base de datos está completamente validada, lo que significa que se conoce la localización del nódulo, la edad del paciente, sexo y diagnóstico.

En esta sección se presentan los resultados experimentales obtenidos con el algoritmo desarrollado. En primer lugar se presentan los resultados al aplicar la segmentación propuesta a la 
totalidad de imágenes de la base de datos JSRT. Para evaluar la efectividad de la segmentación pulmonar, se usó la base de datos libre SCR [20](Segmentation in Chest Radiographs) como referencia estándar que consta de imágenes binarias de los pulmones, segmentadas manualmente por expertos, a partir de la base de datos JSRT. Esta base de datos fue desarrollada por Bram Van Ginneken y su equipo de trabajo, del Images Sciences Institute localizado en el Centro Médico Universitario de Utrecht en los Países Bajos.

Luego se presentan los resultados de los candidatos a nódulo con la segmentación hecha con la base de datos SCR y con la segmentación propuesta para efectos de comparación.

\subsection{Resultados de la segmentación del área pulmonar}

Para evaluar la eficiencia del algoritmo de segmentación del área pulmonar propuesto, se usaron gráficas ROC empleando como estándar de oro la base de datos SCR. Se calcula el número de píxeles que coinciden con la región segmentada y la de referencia, denominados VP (Verdaderos Positivos); los píxeles que por segmentación no corresponden a la referencia pero que se detectan como tal, en este caso se consideran FP (Falsos Positivos); de igual manera los píxeles donde la segmentación no tomó partes que en realidad si pertenecen a la referencia se denominan FN (Falsos Negativos) y por último los VN (Verdaderos Negativos) hacen referencia a los píxeles correctamente clasificados como fondo. Para este efecto se definen las siguientes variables:

$$
\begin{gathered}
\mathrm{PPV}=\frac{\mathrm{VP}}{\mathrm{VP}+\mathrm{FP}} \\
\mathrm{SE}=\frac{\mathrm{VP}}{\mathrm{VP}+\mathrm{FN}} \\
\mathrm{D}=\sqrt{(1-\mathrm{SE})^{2}+(1-\mathrm{PPV})^{2}}
\end{gathered}
$$

$\mathrm{PPV}^{1}$ hace referencia al valor predictivo del resultado positivo o índice de precisión que puede definirse como la proporción de píxeles o elementos detectados correctamente con respecto a todos los elementos detectados. $\mathrm{SE}^{2}$ hace referencia a la sensitividad, la razón de los píxeles detectados correctamente con respecto a todos los píxeles que conforman la referencia (la región pulmonar).

En la gráfica ROC, SE y PPV son los ejes $x$ e y respectivamente y representa la relación entre VP, FN y FP. En la Figura 10 se representa la gráfica ROC generada por las 154 imágenes de prueba con nódulos pulmonares. El mejor método posible de predicción se situaría en un punto en la esquina superior derecha o coordenada $(1,1)$ (clasificación perfecta), representando

\footnotetext{
${ }^{1}$ Predictive Positive Value

${ }^{2}$ Sensitivity
} 
un $100 \%$ de SE (ningún FN) y un $100 \%$ de razón de éxitos (ningún FP). La variable D es la distancia euclidiana desde un punto ubicado sobre la curva hasta la coordenada $(1,1)$.

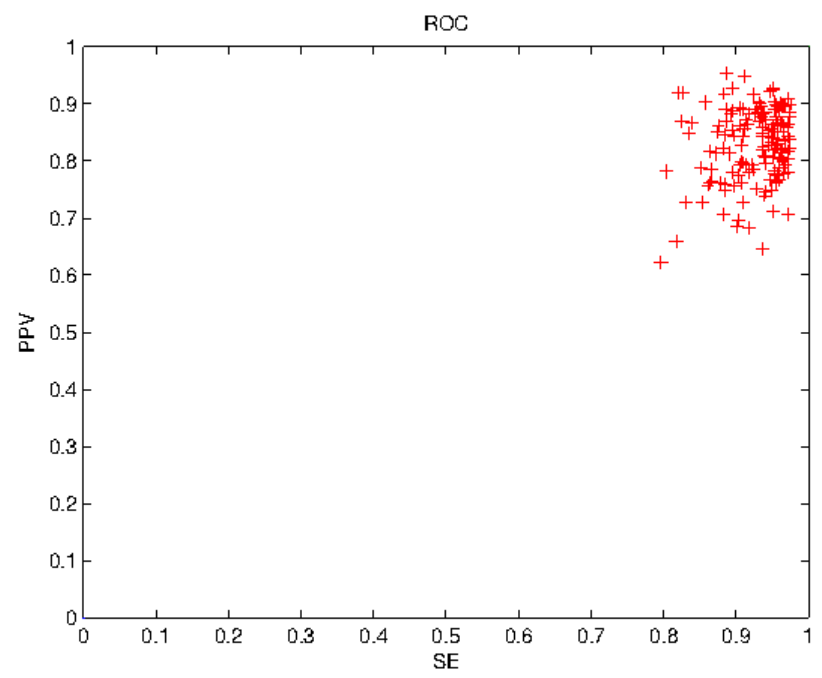

Figura 10. Gráfica ROC generada por las 154 imágenes con nódulo.

Otras medidas son la Especificidad y la Precisión que se miden por medio de las siguientes ecuaciones:

$$
\text { Precisión }=\frac{\mathrm{VP}+\mathrm{VN}}{\mathrm{VP}+\mathrm{VN}+\mathrm{FP}+\mathrm{FN}}
$$

$$
\text { Especificidad }=\frac{\mathrm{VN}}{\mathrm{VN}+\mathrm{FP}}
$$

La precisión es la razón entre los píxeles correctamente clasificados como pulmón y fondo con respecto a la imagen total. La especificidad hace referencia a la probabilidad de clasificar correctamente los píxeles que no corresponden a la región pulmonar.

En la Tabla I se muestran los resultados finales de la segmentación pulmonar de acuerdo a las variables mencionadas.

Tabla I. Valores de la gráfica ROC

\begin{tabular}{cc}
\hline Variable & Valor promedio \\
\hline D & 0.2035 \\
PPV & $81.6 \%$ \\
SE & $92.52 \%$ \\
Precisión & $91.22 \%$ \\
Especificidad & $90.49 \%$ \\
\hline
\end{tabular}




\subsection{Resultados de los candidatos a nódulo}

Todos los filtros de convergencia tienen como parámetro el valor del radio en píxeles de la región de apoyo que se aplica. Sin embargo, no es fácil deducir un solo valor de radio que resalte las regiones nodulares para cada uno de los cuatro filtros. Por tal motivo se hace necesario buscar el valor de radio que genere la mayor detección de las áreas nodulares en la totalidad de las imágenes con nódulo a tratar. Esto fue medido por medio del número de candidatos promedio de nódulos por imagen y la cantidad de nódulos verdaderos detectados, para cada uno de los filtros de convergencia.

Se utilizaron 98 imágenes de la base de datos que corresponden a los niveles 3 , 4 y 5 de sutileza en detección, descartando las dos imágenes que tienen el nódulo fuera del área pulmonar.

Como el desarrollo del sistema utiliza los filtros de convergencia dos veces, para la imagen original y para la de la resta contralateral, entonces se debe buscar para cada filtro el radio de mayor detección y en las dos condiciones mencionadas. En la Tabla II se muestran los radios de mayor detección para cada filtro, con las dos condiciones y con el número de candidatos en promedio por imagen.

Tabla II. Valores de radio para los cuales se presentó la mayor detección

\begin{tabular}{ccccccc}
\hline \multirow{2}{*}{ Filtro } & \multicolumn{3}{c}{ Imagen original } & \multicolumn{3}{c}{ Imagen contralateral } \\
\cline { 2 - 7 } & Radio & Candidatos & $\%$ de detección & Radio & Candidatos & $\%$ de detección \\
\hline Filtro de moneda & 15 & 27.88 & $73.43 \%$ & 19 & 36.56 & $73.47 \%$ \\
\hline Filtro de iris & 8 & 49.79 & $68.37 \%$ & 11 & 59.75 & $71.43 \%$ \\
\hline Filtro de anillo adaptativo & 12 & 30.52 & $73.47 \%$ & 12 & 43.84 & $84.69 \%$ \\
\hline Filtro de banda deslizante & 8 & 42.33 & $79.59 \%$ & 16 & 44.08 & $88.78 \%$ \\
\hline
\end{tabular}

Al aplicar el algoritmo de la Figura 4 con los radios indicados en la Tabla II se obtiene una reducción significativa de candidatos a nódulo como se observa en la Tabla III. Se puede observar que el filtro de moneda y el filtro de anillo presentaron una alta tasa de detección con un número bajo de candidatos. Cuando se usa la segmentación propuesta el filtro de moneda y el filtro de anillo adaptativo obtuvieron una tasa de detección de $68.37 \%$ y $70.41 \%$ respectivamente.

Tabla III. Resultado de los filtros de convergencia

\begin{tabular}{ccc}
\hline Tipo de filtro & Candidatos & \% de detección \\
\hline Filtro de moneda & 12.26 & $74.48 \%$ \\
Filtro de iris & 30.01 & $62.24 \%$ \\
Filtro de anillo adaptativo & 12.73 & $75.51 \%$ \\
Filtro de banda deslizante & 27.6 & $78.57 \%$ \\
\hline
\end{tabular}

Como estos dos filtros fueron los más eficientes en términos cuantitativos, una comparación adicional se muestra en la Tabla IV, donde se muestra el número de candidatos promedio por imagen en las 98 imágenes y las 93 imágenes sin nódulo. 
Tabla IV. Comparación del número de candidatos promedio en los filtros de moneda y anillo

\begin{tabular}{ccccc}
\hline Tipo de filtro & \multicolumn{2}{c}{ Segmentación manual (SCR) } & \multicolumn{2}{c}{ Segmentación propuesta } \\
\cline { 2 - 5 } & Imágenes con nódulo & Imágenes sin nódulo & Imágenes con nódulo & Imágenes sin nódulo \\
\hline Filtro de moneda & 12.26 & 11.26 & 15.62 & 14.87 \\
Filtro de anillo adaptativo & 12.73 & 12.13 & 16.51 & 16.25 \\
\hline
\end{tabular}

En la Figura11 se aprecian los resultados de la aplicación de los filtros de convergencia en imágenes de la base de datos. Se observa como lo filtros de moneda y anillo generan un realce más notorio comparado con los filtros restantes de los nódulos pulmonares. Dicha Figura puede ser comparada con los resultados obtenidos en [15] donde se usa el filtro LoG como método de realce de los nódulos. Los filtros de convergencia de anillo y moneda realzan los candidatos a nódulo con un mayor contraste con respecto a los filtros LoG.
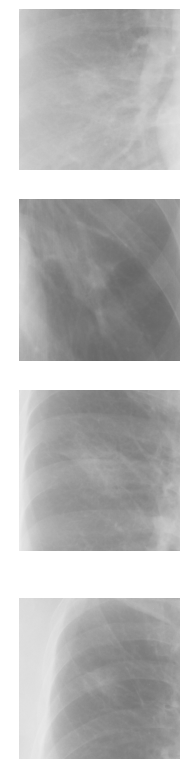

(a)
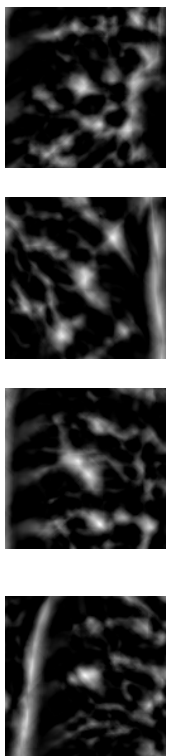

(b)
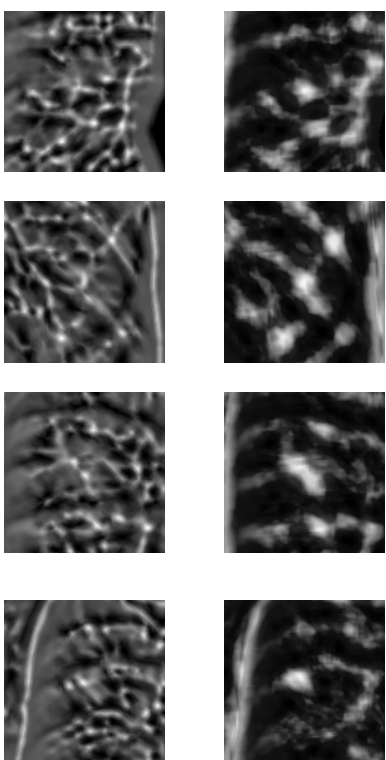

(c)
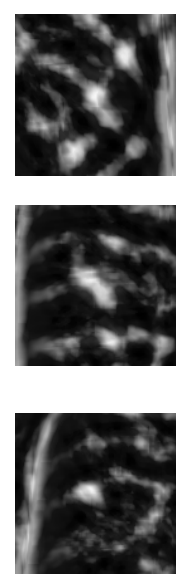

(d)
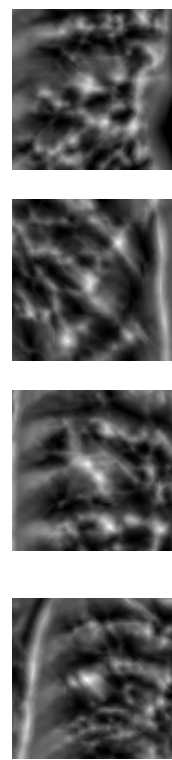

(e)

Figura 11. Comparación de imágenes con los filtros de convergencia: (a) Imagen original, (b) resultado del filtro de moneda, (c) resultado del filtro de iris, (d) resultado del filtro de anillo adaptativo y (e) resultado del filtro de banda deslizante

\section{Discusión}

La base de datos JSRT fue recolectada de diferentes instituciones médicas, de manera que incluye un amplio rango de características de las radiografías, como la distancia de toma, nivel de iluminación, sexo, edad del paciente, entre otras. A pesar de eso, la segmentación propuesta funciona para todas las imágenes propuestas, con una alta sensitividad y precisión. Como el método desarrollado no necesita el conocimiento a priori de la forma de los pulmones y trabaja en forma automática, logrando segmentar el área pulmonar de las imágenes tratadas, 
se considera valioso frente a otras técnicas expuestas en la introducción, ya que no requiere de etapas de entrenamiento como los enfoques más usados en este campo: redes neuronales o inteligencia artificial.

En el algoritmo de realce de los nódulos, el filtro de moneda y el filtro de anillo adaptativo, que son los que tienen claramente marcada una región de apoyo circular, fueron los más eficientes al presentar una tasa de detección alta con menor número de candidatos. Por el contrario, el filtro de iris, a pesar de que presenta una tasa de detección alta, el número de candidatos igualmente es alto. Sin embargo, como las tasas de detección se tomaron al final del proceso, claramente pueden ser sesgadas por el método de binarización. Usando el filtro de moneda y el filtro de anillo adaptativo en la segmentación propuesta se evidenció un mayor número de candidatos y una disminución leve en la tasa de detección.

\section{Conclusiones}

Para el realce de los candidatos a nódulo pulmonar se implementaron los filtros de convergencia con el fin de que las regiones circulares se pudieran destacar con respecto al resto de la imagen. De la Tabla II, se puede concluir que para cada tipo de filtro hay un valor de radio diferente que genera la mayor tasa de detección. Esto aplica tanto para las imágenes originales como para las contralaterales.

Existen las técnicas de resta temporal, que se basa sólo en la diferencia temporal entre dos imágenes y resta de doble exposición basada en la diferencia de la toma de dos imágenes, variando la energía de los rayos X entre una y otra. Al contar con una radiografía por paciente, se empleó la resta contralateral la cual permite eliminar regiones óseas y resaltar nódulos pulmonares.

Los resultados comparados con los trabajos referenciados, en general fueron satisfactorios por cuanto se pudo evidenciar que al aplicar los filtros de convergencia los nódulos pulmonares se resaltan. Como estos filtros se adaptan a formas circulares, son una alternativa en la detección de nódulos. El método no requirió de conocimiento a priori lo cual denota una ventaja al adaptarse a los distintos tipos de radiografía de tórax, como lo realizado por varios autores y por otras técnicas [24] [23][2][6] [15] [10][19]. Estos métodos alcanzaron una tasa de detección del $70 \%$ al $90 \%$ y entre 2 y 14 falsos positivos por imagen, pero algunos usaron sus propias bases de datos.

La meta de obtener mayores tasas de detección con la disminución del mayor número de falsos positivos por imagen es un objetivo aún común en diferentes centros de investigación internacionales. Para un trabajo futuro se recomienda la implementación de otros criterios de clasificación adicionales que permitan disminuir el número de candidatos por imagen, ya que en este trabajo solo se utilizaron tres características geométricas como son la excentricidad, la redondez y el área. Se pueden utilizar otros criterios, variables o características como la entropía, centro de masas, desviación estándar, entre muchas otras. También se puede extender 
el uso de las imágenes de la base de datos a los niveles 1 y 2 de sutileza, así como las que tienen nódulos que se encuentran en las regiones escondidas de la región pulmonar.

\section{Agradecimientos}

Los autores desean agradecer a la JSRT por facilitar la base de datos de las radiografías de tórax. Igualmente al doctor Bram Van Ginneken y colaboradores por las segmentaciones manuales de los pulmones usadas en este artículo.

\section{Referencias}

[1] P. Meza Conejo and K. Gutreiman Rubinstein. Nódulo pulmonar solitario: Revisión bibliográfica. Revista Médica de Costa Rica y Centro América, LXIV(580):155-159, 2007.

[2] G. Coppini, S. Diciotti, M. Falchini, N. Villari, and G. Valli. Neural Networks for Computer-Aided Diagnosis: Detection of Lung Nodules in Chest Radiograms. IEEE transactions on information technology in biomedicine: a publication of the IEEE Engineering in Medicine and Biology Society, 7(4):344-57, 2003.

[3] O. Demirkaya, M. H. Asyali, and P. K. Sahoo. Image Processing with Matlab: Applications in Medicine and Biology, pages 260-261. CRC Press, 2009.

[4] T. Esteves, P. Quelhas, A. M. Mendonça, and A. Campilho. Gradient convergence filters and phase congruency approach for in vivo cell nuclei detection. Machine Vision and Applications, 23:623-638, 2012.

[5] M. Gabrielli. Nódulo pulmonar solitario: Desafío diagnóstico y terapéutico (Artículo de actualización). Cuadernos de cirugía (Valdivia), 21:65-74, 2007.

[6] T. Hara, H. Fujita, and Jing Xu. Development of automated detection system for lung nodules in chest radiograms. Proceedings Intelligent Information Systems, IIS:71-74, 1997.

[7] T. Kawaguchi, Y. Harada, R. Nagata, and H. Miyake. Image registration methods for contralateral subtraction of chest radiographs. 2010 3rd International Conference on Biomedical Engineering and Informatics, (Bmei):306-313, 2010

[8] T. Kawaguchi, R. Nagata, Y. Harada, and H. Miyake. Image registration using b-splines for contralateral subtraction of chest radiographs. TENCON 2010 - 2010 IEEE Region 10 Conference, pages 1589-1594, 2010.

[9] N. Otsu. A Threshold Selection Method from Gray-Level Histograms. IEEE Transactions on Systems, Man, and Cybernetics, SMC-9(1):62-66, 1979.

[10] E. Casiraghi P. Campadelli and D. Artioli. A Fully Automated Method for Lung Nodule Detection From Postero-Anterior Chest Radiographs. IEEE transactions on medical imaging, 25(12):1588-1603, 2006.

[11] C. J. Vicente Peña. AHE (Ecualización del Histograma Adaptativo), 1998.

[12] N. Petkov and P. Kruizinga. Computational models of visual neurons specialised in the detection of periodic and aperiodic oriental simuli: bar and gratings cells. Biological Cibernetics, 76:83-96, 1997.

[13] E. Pietka and O. Ratib. Segmentation of chest radiographs. Proceedings of the Annual International Conference of the IEEE Engineering in Medicine and Biology Society, pages 1911-1912, 1992.

[14] F. M. Rodríguez and F. J. Suárez López. Identificación dactilar basada en filtros Gabor. Universidad de Vigo URSI, 2004.

[15] Z. Shi, J. Bai, L. He, T. Nakamura, Q. Yao, and H. Itoh. A Method for Enhancing Lung Nodules in Chest Radiographs by Use of LoG Filter. 2009 2nd International Congress on Image and Signal Processing, pages $1-4,2009$. 
[16] J. Shiraishi, S. Katsuragawa, J. Ikezoe, T. Matsumoto, T. Kobayashi, K. Komatsu, M. Matsui, H. Fujita, Y. Kodera, and K. Doi. Development of a Digital Image Database for Chest Radiographs With and Without a Lung Nodule: Receiver Operating Characteristic Analysis of Radiologists' Detection of Pulmonary Nodules. American Journal of Roentgenology, 174:71-74, 2000.

[17] E. Soleymanpour, H. R. Pourreza, E. Ansaripour, and M. S. Yazdi. Fully Automatic Lung Segmentation and Rib Suppression Methods to Improve Nodule Detection in Chest Radiographs. Journal of Medical Signals and Sensors, 1(3):191-199, 2011.

[18] M. Souto, P.G. Tahoces, J.J. Suárez, M.J. Lado, M. R. Jardin, J. Remy, and J.J. Vidal. Detección automática de nódulos pulmonares en tomografía computarizada. Un estudio preliminar. Radiología, 50(5):387-392, 2008.

[19] S. Tsukuda, A. Heshiki, S. Katsugarawa, Q. Li, H. MacMahon, and K. Doi. Detection of Lung Nodules on Digital Chest Radiographs: Potential Uselfuness of a New Contralateral Subtraction Tecnique. Radiology, 223:199-203, 2002.

[20] B. van Ginneken, M. B. Stegmann, and M. Loog. Segmentation of anatomical structures in chest radiographs using supervised methods: a comparative study on a public database. Medical Image Analysis, 10(1):19-40, 2006.

[21] B. van Ginneken, B. M. ter Haar Romeny, and M. Viergever. Computer-Aided Diagnosis in Chest Radiography: A Survey. IEEE Transactions on Medical Imaging, 20(12):1228-1241, 2001.

[22] J. Wei, Y. Hagihara, and H. Kobatake. Detection of Cancerous Tumors on Chest X-ray Images Candidate Detection Filter and Its Evaluation. Proceedings of International Conference on Image Analysis and Processing (ICIP), pages 397-401, 1999.

[23] H. Yoshida, S. Katsuragawal, and K. Doit. Wavelet snake for classification of nodules and false positives in digital chest radiographs. Proceedings 19th International Conference IEEE EMBS, 509(C):1-3, 1997.

[24] H. Yoshida, B. Keserci, K. Doi, and N. Chicago. Computer-aided diagnosis of pulmonary nodules in chest radiographs: A wavelet-based snake approach.

[25] P. Yu, J. Zhao, H. Xu, C. Yang, X. Sun, S. Chen, and L. Mao. Computer Aided Detection for Pneumoconiosis Based on Histogram Analysis. 2009 First International Conference on Information Science and Engineering, pages 3625-3628, 2009.

\section{Julián Mauricio Aguillón Pérez}

Nació en Duitama, Colombia. Ingeniero Electrónico de la Universidad Distrital Francisco José de Caldas. Sus áreas de interés son el Procesamiento Digital de Imágenes, las Telecomunicaciones y la Nanotecnología.

\section{Sergio Alejandro Duarte Pacheco}

Nació en Bucaramanga, Colombia. Ingeniero Electrónico de la Universidad Distrital Francisco José de Caldas. Sus áreas de interés son el Procesamiento Digital de Señales, la Nanotecnología, la Telemática y las Telecomunicaciones.

\section{Rodrigo Javier Herrera García}

Nació en Bogotá, Colombia. Obtuvo su título como Ingeniero Electrónico en 1987 y como Magíster en Teleinformática en 1999 en la Universidad Distrital Francisco José de Caldas. Se ha desempeñado como docente universitario desde 1988 en las Universidades Santo Tomás de Aquino y Distrital de Bogotá en las áreas de sistemas digitales y electrónica analógica. A partir de 1997 se vinculó como profesor de tiempo completo en la Facultad de Ingeniería de la Universidad Distrital Francisco José de Caldas. Actualmente, su área de interés es el procesamiento digital de imágenes y es director del grupo de Investigación, Desarrollo y Aplicaciones en Señales IDEAS-UD. 